\title{
An Efficient Scheme for the Single Source Shortest Path Problem based on Dijkstra and SPFA Methodologies
}

\author{
G. L. Prajapati, PhD \\ Dept. of Comp. Engg. \\ IET-Devi Ahilya University
}

Indore

\author{
Pulkit Singhal \\ Dept. of Comp. Engg. \\ IET-Devi Ahilya University \\ Indore
}

\author{
Ayush Ranjan \\ Sharma \\ Dept. of Comp. Engg. \\ IET-Devi Ahilya University \\ Indore
}

\author{
Neelesh Chourasia \\ Dept. of Comp. Engg. \\ IET-Devi Ahilya University \\ Indore
}

\begin{abstract}
This paper presents detailed comparisons and analysis of various single source shortest path algorithms. The paper proposes comparison among these algorithms on the basis of execution time taken by the algorithms to completely find the shortest path to all the nodes from a starting node. The algorithms have been analyzed on the various parameters: number of vertices, number of edges, and structure of the graph. This analysis will help in selecting the appropriate algorithm to be used in solving a particular real-life problem. This paper also proposes an algorithm that works efficiently over all types of the graph.
\end{abstract}

\section{General Terms}

Shortest Path, Algorithms, Theoretical Computer Science.

\section{Keywords}

Single Source Shortest Path, Execution Time, Performance Analysis

\section{INTRODUCTION}

Shortest path problem [1] is the problem of finding a path between two vertices in a graph such that the sum of the weights of its constituent edges is minimized. Without the use of shortest path algorithms, the naïve approach for finding the shortest path between two vertices is to enumerate all possible paths between the vertices and select the shortest one i.e. brute force. However for the various domain specific applications of the shortest path problem, brute force approach is not feasible and hence require more optimal solution i.e. the shortest path algorithms. The aim of shortest path algorithms is to find the shortest path among all paths available between the pair of vertices. Some shortest path algorithms works only over the non-negative weighted graphs while some can works with negative weighted graphs too. Also the distinction between the algorithms is made by whether these are single source shortest path (SSSP) or all pair shortest path algorithm (APSP). This paper focuses on single source shortest path algorithms for the non-negative weighted graphs. Some of the shortest path algorithms are greedy in nature while some uses the dynamic programming. The shortest path algorithms works over the principle of relaxation. In such algorithms, optimization is based on the number of times relaxation is performed during the execution. Various real life applications of shortest path algorithms are finding the shortest route between the two places, social network analysis (SNA) to calculate degree of separation between two users on a social networking medium and so on. This paper compares the set of algorithms among themselves on the basis of execution time on datasets of different types.

\section{SET OF SHORTEST PATH ALGORITHMS UNDER ANALYSIS}

\subsection{Bellman-Ford Algorithm}

Bellman-Ford algorithm [2] uses the principle of edge-based relaxation. In every iteration it relaxes all the edges and these iterations are done $\mathrm{V}-1$ times as the maximum number of edges in the shortest path between two vertices are V-1, where $\mathrm{V}$ is the number of vertices in the graph. If the relaxation can be done more than V-1 times, it indicates the presence of the negative cycles. The worst case complexity of this algorithm is $\mathrm{O}(\mathrm{VE})$.

\subsection{Dijkstra Algorithm}

Dijsktra algorithm [3] uses the same principle of relaxation as Bellman-Ford algorithm. It works over the graph with nonnegative weighted edges only. In every iteration, it greedily chooses the vertex which is not selected before and has minimum cost. It tries to relax vertices through the selected vertex. Selection of vertex with minimum cost primarily affects the complexity of algorithm. In case of binary heap, worst case complexity of this algorithm is $\mathrm{O}(\mathrm{E} \log \mathrm{V})$. We are also considering an implementation of Dijkstra [4] which fastens the performance in case where the number of distinct weighted edges is less.

\subsection{Pape-Levit Algorithm}

Pape-Levit [5] is an incremental graph algorithm, where the two sets of vertices are maintained. One set of vertices contains those vertices which are scanned at least once while the second set contains those vertices which have never been scanned. The priority is given to first set for selection of vertex. The worst case complexity of this algorithm is $\mathrm{O}(\mathrm{VE})$. It works quite fast on the randomly weighted graphs.

\subsection{SPFA}

The Shortest Path Faster Algorithm (SPFA) [6] is an improvement of the Bellman-Ford algorithm which computes single-source shortest paths in a weighted directed graph. The algorithm is believed to work well on random sparse graphs and is particularly suitable for graphs that contain negativeweight edges. The performance of the algorithm is strongly determined by the order in which candidate vertices are used to relax other vertices.

\subsection{Proposed Algorithm}

As the performance of some algorithms highly depends over the order in which candidate vertices are used to relax the other vertices, we can see the linear time performance over the various type of graph structures while it also goes to $O(V E)$ worst case time complexity. On the other hand, algorithms like the Dijkstra gives $\mathrm{O}(\mathrm{E} \log \mathrm{V})$ time complexity on every type of graph structure. This proposed algorithm harnesses the 
power of both the algorithms. Proposed solution uses SPFA upto a certain limit of operations and if the shortest path is not found it moves to Dijkstra algorithm and the intermediate information produced by SPFA can be used to fasten the computation of Dijkstra algorithm. Let the transition factor be $\mu$. Proposed solution uses SPFA till $\mu^{*}(\mathrm{~V}+\mathrm{E})$ operations, after this it transits to Dijkstra. The value of transition factor $(\mu)$ will decide how fast the transition between these two algorithms happens. This algorithm will reduce the average time for finding the shortest path on a general graph.

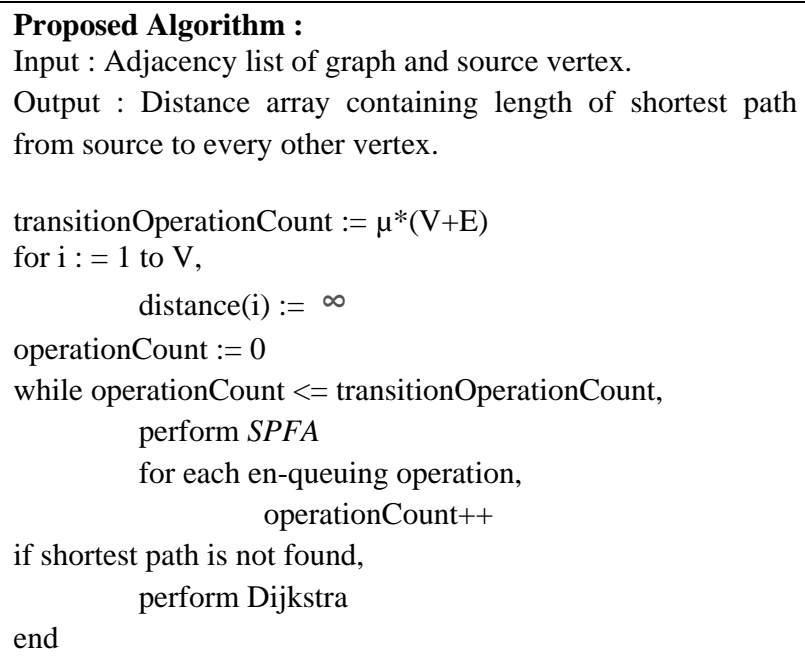
incrementing value of $\mathrm{x}$ by 1 . This algorithm has an important factor $(\mu)$ which we termed as the transition factor. This transition factor decides how fast it moves from SPFA to the Dijkstra. If this factor is small then it moves to the Dijkstra quite fast, but if this factor is high it ends up using SPFA all the time. So a moderate value is required for this factor. Our algorithm runs SPFA algorithm for the $\mu^{*}(\mathrm{~V}+\mathrm{E})$ operations, after that if it does not able to find the shortest path it moves to the Dijkstra algorithm. In best case it finds the shortest path in the time of SPFA itself, while in the worst case it ends up using both the algorithms. So asymptotically best case of this proposed algorithm is $\mathrm{O}\left(\mu^{*}(\mathrm{~V}+\mathrm{E})\right)$ while worst case is $\mathrm{O}((\mathrm{V}+\mathrm{E}) *(\log \mathrm{V}+\mu))$. In general case it is assumed that $\mu$ is a small constant value. In the experimental analysis three different values of $\mu$ are considered.

\section{ANALYSIS}

\subsection{Experiment Specifications}

The tests were run on Intel Core i33217U @1.80 GHz CPU (CPU family 6, Model A, Stepping 9). Number of CPU(s) and socket is 1 having 2 Thread(s) per core and 2 Core(s) per socket. The algorithms were tested in presence of $64 \mathrm{~K} \mathrm{L1d}$ cache, $64 \mathrm{~K}$ of L1i cache, $512 \mathrm{~K}$ of L2 cache, $3072 \mathrm{~K}$ of L3 cache, and 4GB DDR3 RAM. The system had Intel HD 4000 Graphics with $349 \mathrm{MHz}$ GPU clock. Byte Order of CPU used is Little Endian. The computer was running Windows 10 64bit. All programs were written in $\mathrm{C}++$ programming language with $\mathrm{g}++$ compiler 4.8 .4 producing x 86 _64 "64-bit" code.

\subsection{Analysis Specification}

The types of datasets used for the analysis of algorithms were: Some random datasets that were generated using the Prüfer sequence [7] consisting of various vertices and edge count. With the use of Prüfer sequence [7], generation of test data for trees can be done in equi-probable manner. In the random datasets we generated different structures like trees and graph, having vertices count from 10,000 to $10,000,000$. For complete graphs, vertices count is varied from 100 to 5000 . Along with random graph structures, some special graphs [8] are also generated which lead to the worst case for various algorithms. Experiments uses some datasets from DIMACS Implementation Challenge - Shortest Paths [9] (Northeast USA) containing 1,524,453 nodes and 3,897,636 edges. Along with this datasets from Stanford Large Network Dataset [10] Collection were included; one is the YouTube online social network containing $1,134,890$ vertices and 2,987,624 edges, other one is the Amazon product copurchasing network from June 12003 containing 403,394 vertices and 3,387,388 edges. The analysis report is based on: the execution time taken by algorithms on all the above mentioned datasets.

\section{EXPERIMENTAL RESULTS}

This analysis report is based on the execution time taken by algorithms on all the above mentioned parameters working with the above mentioned datasets. The resultant execution time is calculated as shown in Table 1 along with its graphical representation in Figure 1. Datasets were taken from 9th DIMACS implementation: shortest path - Northeast USA, Stanford large network dataset collection - YouTube community, Amazon sales co-purchasing network from June $1,2003$.

\subsection{Analysis on Trees}

Table 1 shows analysis of various algorithms on trees with different number of nodes. Figure 1 shows graph of execution time on different number of vertices in tree.

\subsection{Analysis on Randomistic graphs}

Table 2 shows analysis of various algorithms on randomistic graphs with different number of nodes and edges.

\subsection{Analysis on Complete graphs}

Table 3 shows analysis of various algorithms on complete graphs with different number of nodes. Figure 2 shows graph of execution time on different number of vertices in complete graph.

\subsection{Analysis on Special graphs}

Table 4 shows analysis of various algorithms on Special graphs with different number of nodes. Figure 3 shows graph of execution time on different number of vertices in special graph.

\subsection{Analysis on Benchmarking Datasets}

Table 5 shows analysis of various algorithms on benchmarking graphs. Figure 4 shows execution time on DIMAC distance based dataset. Figure 5 shows execution time on DIMAC time based dataset. Figure 6 shows execution time on Amazon co-purchasing dataset. Figure 7 shows execution time on YouTube community dataset. 
Table 1: Showing the execution time of the algorithms on tree Set of algorithms

\begin{tabular}{|c|c|c|c|c|c|c|c|c|}
\hline & $\begin{array}{l}\text { Bellman- } \\
\text { Ford }\end{array}$ & $\begin{array}{l}\text { Dijkstra } \\
\text { (Binary } \\
\text { Heap) }\end{array}$ & $\begin{array}{l}\text { Dijkstra } \\
\text { (Edge } \\
\text { based) }\end{array}$ & $\begin{array}{l}\text { Pape- } \\
\text { Levit }\end{array}$ & SPFA & $\begin{array}{l}\text { Proposed } \\
\text { Algorithm } \\
(\mu=1)\end{array}$ & $\begin{array}{l}\text { Proposed } \\
\text { Algorithm } \\
(\mu=2)\end{array}$ & $\begin{array}{l}\text { Proposed } \\
\text { Algorithm } \\
(\mu=3)\end{array}$ \\
\hline 10000 & 0.0400 & 0.0350 & 0.1380 & 0.0020 & 0.0050 & 0.0060 & 0.0070 & 0.0040 \\
\hline 50000 & 0.4510 & 0.1220 & 0.6020 & 0.0420 & 0.0210 & 0.0260 & 0.0240 & 0.0300 \\
\hline 100000 & 0.7970 & 0.2880 & 0.8540 & 0.0880 & 0.0490 & 0.0560 & 0.0550 & 0.0620 \\
\hline 500000 & 6.6220 & 1.6870 & 3.1460 & 0.3700 & 0.3280 & 0.3490 & 0.3190 & 0.3310 \\
\hline 1000000 & 23.5760 & 3.5230 & 5.8320 & 0.7990 & 0.6770 & 0.7040 & 0.6970 & 0.7230 \\
\hline 5000000 & 80.4500 & 22.8310 & 34.3050 & 5.0920 & 4.2270 & 3.6200 & 4.3780 & 4.5780 \\
\hline 10000000 & - & 50.5150 & - & 13.5350 & 10.9730 & 13.3140 & 11.6240 & 10.6110 \\
\hline
\end{tabular}

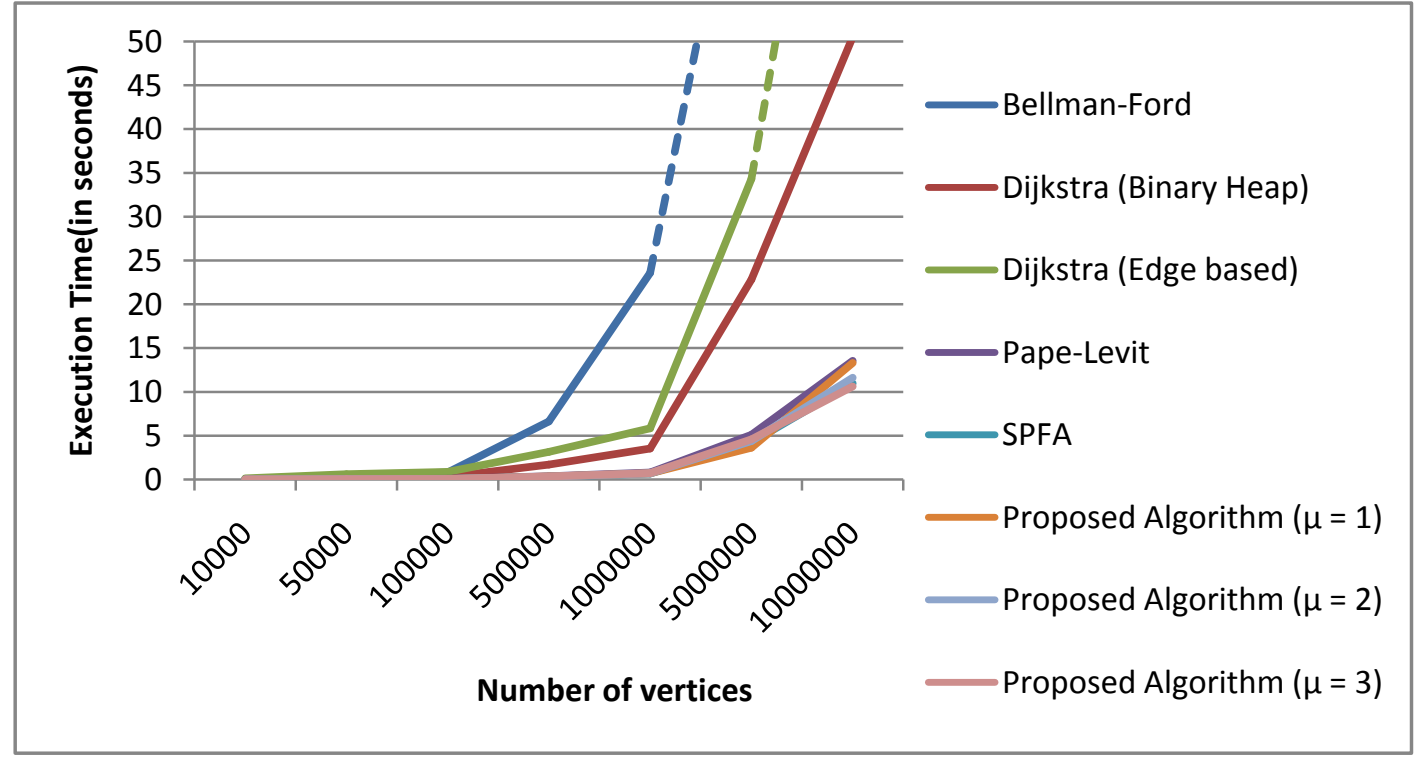

Figure 1: Graph showing execution time vs. number of vertices in tree

Table 2: Showing the execution time of the algorithms on graphs

\begin{tabular}{|c|c|c|c|c|c|c|c|c|c|c|}
\hline & \multicolumn{9}{|c|}{ Set of algorithms } & \multirow[b]{2}{*}{$\begin{array}{l}\text { Proposed } \\
\text { Algorithm } \\
\quad(\mu=3)\end{array}$} \\
\hline \multirow{3}{*}{ 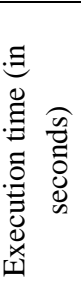 } & $\begin{array}{l}\text { Number } \\
\text { of vertices }\end{array}$ & $\begin{array}{l}\text { Number } \\
\text { of edges }\end{array}$ & $\begin{array}{l}\text { Bellman- } \\
\text { Ford }\end{array}$ & $\begin{array}{c}\text { Dijkstra } \\
\text { (Binary } \\
\text { Heap) }\end{array}$ & $\begin{array}{c}\text { Dijkstra } \\
\text { (Edge } \\
\text { based) }\end{array}$ & $\begin{array}{l}\text { Pape- } \\
\text { Levit }\end{array}$ & SPFA & $\begin{array}{c}\text { Proposed } \\
\text { Algorithm } \\
\quad(\mu=1)\end{array}$ & $\begin{array}{l}\text { Proposed } \\
\text { Algorithm } \\
(\mu=2)\end{array}$ & \\
\hline & 10000 & 30000 & 0.0200 & 0.0770 & 0.3060 & 0.1900 & 0.0370 & 0.1150 & 0.1150 & 0.0850 \\
\hline & 10000 & 40000 & 0.0240 & 0.0750 & 0.2620 & 0.2820 & 0.0300 & 0.0960 & 0.0820 & 0.0730 \\
\hline
\end{tabular}




\begin{tabular}{|c|c|c|c|c|c|c|c|c|c|}
\hline 10000 & 50000 & 0.0310 & 0.0810 & 0.3030 & 0.4980 & 0.0360 & 0.1210 & 0.1060 & 0.1000 \\
\hline 100000 & 300000 & 0.2110 & 0.8330 & 1.7370 & 15.6010 & 0.5730 & 1.2670 & 1.2520 & 1.1550 \\
\hline 100000 & 400000 & 0.2900 & 0.7560 & 1.9650 & 33.4720 & 1.0100 & 1.5580 & 1.6560 & 1.8150 \\
\hline 100000 & 500000 & 0.5030 & 1.0560 & 1.9810 & 33.9610 & 0.8640 & 1.3890 & 1.4270 & 1.3800 \\
\hline 1000000 & 3000000 & 2.2170 & 8.2320 & 13.1330 & - & 8.0270 & 14.5790 & 12.5150 & 15.7310 \\
\hline 1000000 & 4000000 & 4.5570 & 9.2260 & 14.0800 & - & 8.9250 & 15.6860 & 16.8670 & 15.9590 \\
\hline 1000000 & 5000000 & 4.2640 & 10.4900 & 19.8210 & - & 15.2440 & 17.3900 & 13.0030 & 13.3360 \\
\hline
\end{tabular}

Table 3: Showing the execution time of the algorithms on complete graphs

Set of Algorithms

\begin{tabular}{|c|c|c|c|c|c|c|c|c|}
\hline $\begin{array}{l}\text { Number of } \\
\text { Vertices }\end{array}$ & $\begin{array}{l}\text { Bellman- } \\
\text { Ford }\end{array}$ & $\begin{array}{c}\text { Dijkstra } \\
\text { (Binary } \\
\text { Heap) }\end{array}$ & $\begin{array}{c}\text { Dijkstra } \\
\text { (Edge } \\
\text { based) }\end{array}$ & Pape-Levit & SPFA & $\begin{array}{l}\text { Proposed } \\
\text { Algorithm } \\
(\mu=1)\end{array}$ & $\begin{array}{l}\text { Proposed } \\
\text { Algorithm } \\
(\mu=2)\end{array}$ & $\begin{array}{l}\text { Proposed } \\
\text { Algorithm } \\
\quad(\mu=3)\end{array}$ \\
\hline 300 & 0.0160 & 0.0000 & 0.0160 & 0.0620 & 0.0310 & 0.0160 & 0.0000 & 0.0000 \\
\hline 600 & 0.0310 & 0.0160 & 0.0780 & 0.7500 & 0.1100 & 0.0320 & 0.0250 & 0.0250 \\
\hline 1000 & 0.1090 & 0.0510 & 0.2190 & 5.0910 & 0.5320 & 0.1530 & 0.1150 & 0.1150 \\
\hline 3000 & 2.0720 & 0.4870 & 2.8640 & 134.8480 & 6.5170 & 0.5060 & 0.8270 & 0.8270 \\
\hline 5000 & 4.8120 & 0.7860 & 5.9740 & - & 16.2580 & 1.8700 & 2.2150 & 2.2150 \\
\hline
\end{tabular}

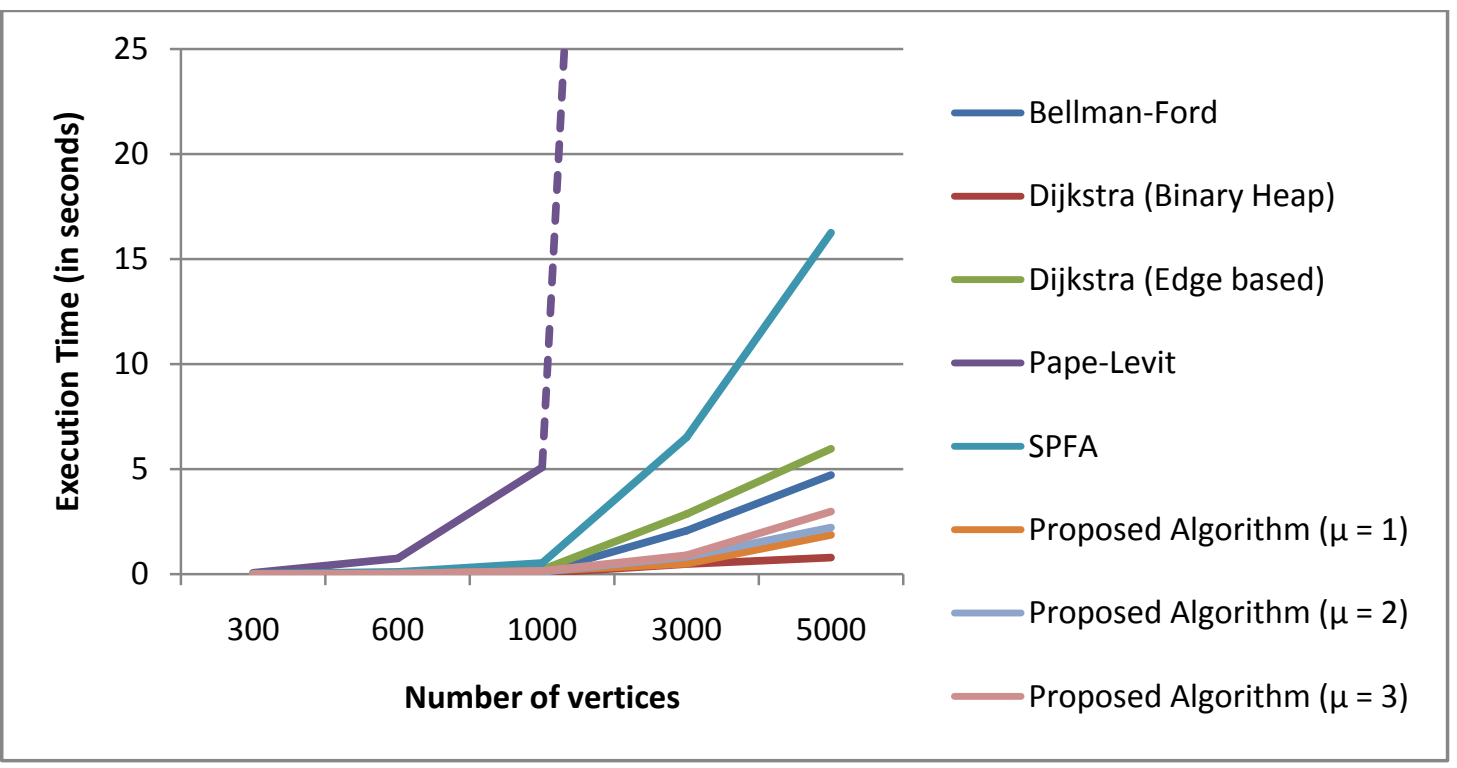

Figure 2: Graph showing execution time vs. number of vertices in complete graph 
Table 4: Showing the execution time of the algorithms on special graphs

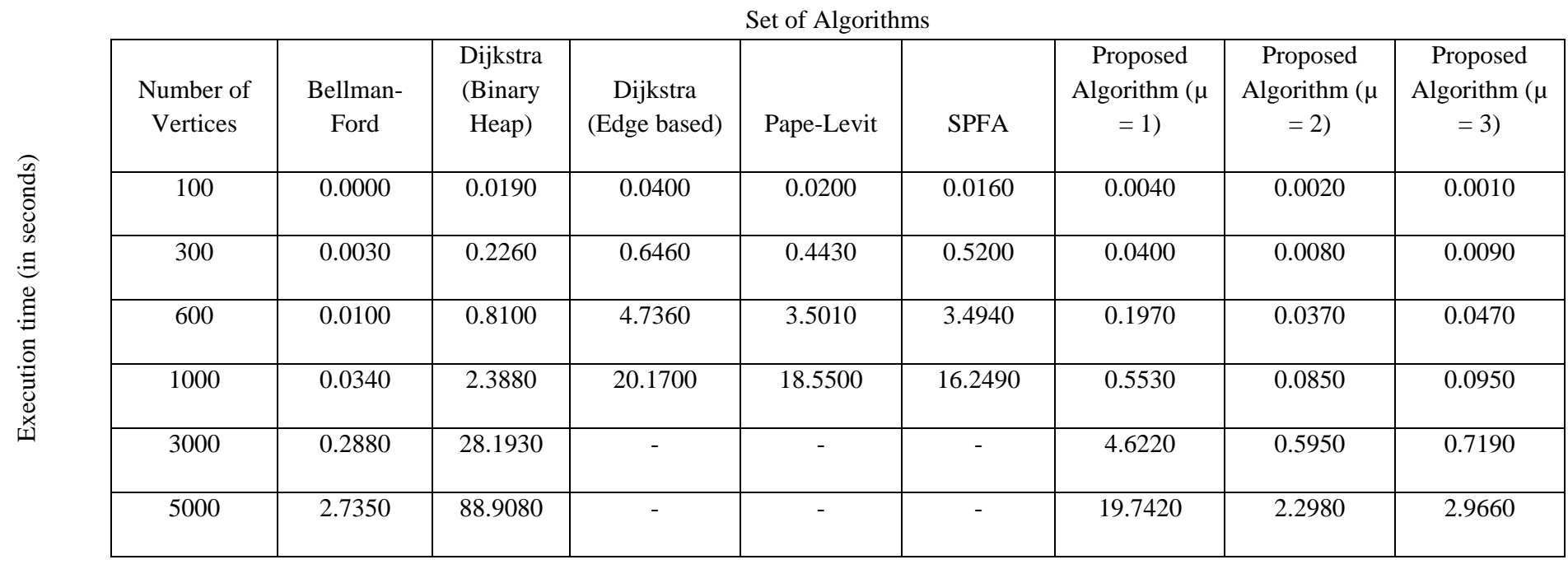

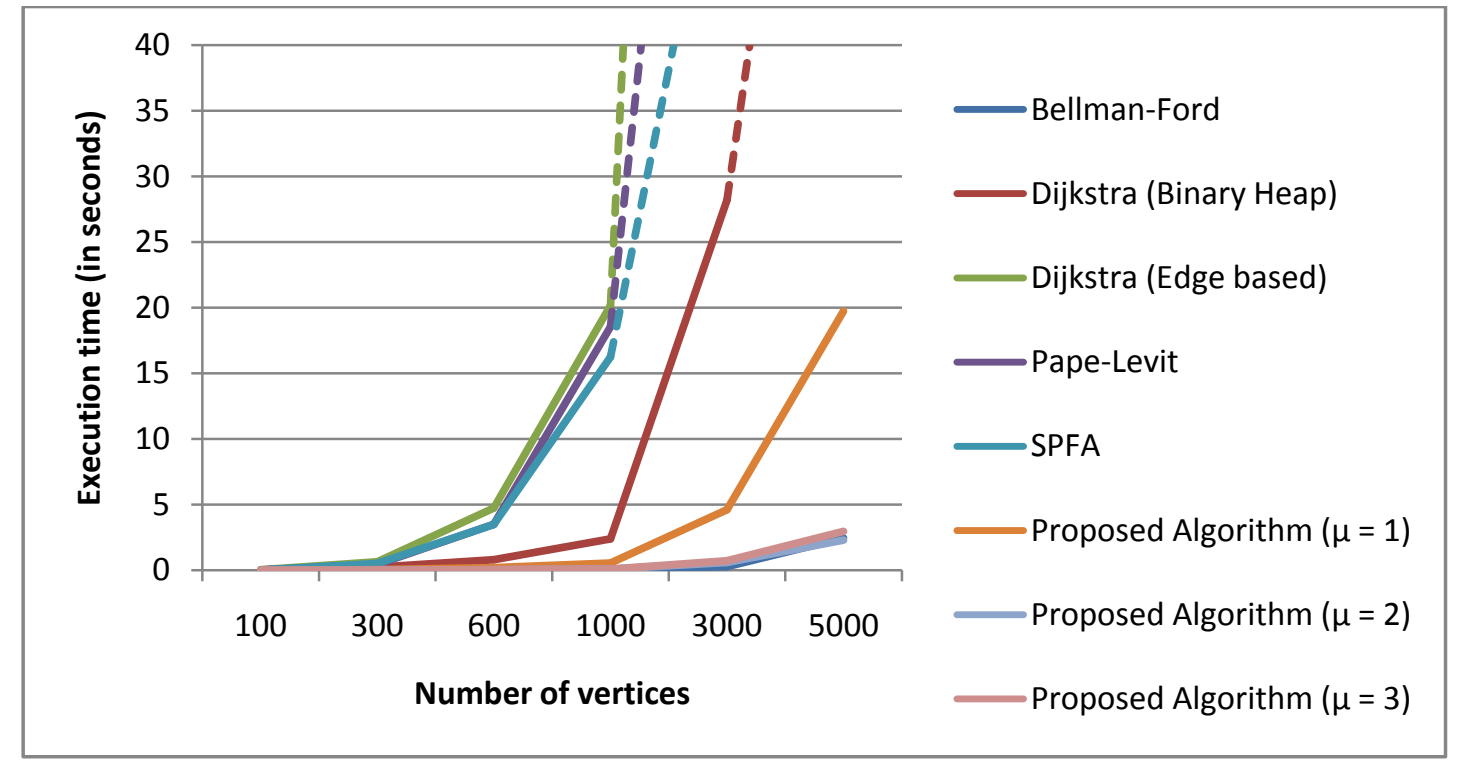

Figure 3: Graph showing execution time vs. number of vertices in special graph

Table 5: Showing the execution time of the algorithms on benchmarking datasets

\begin{tabular}{|c|c|c|c|c|c|c|c|c|}
\hline & \multicolumn{8}{|c|}{ Set of algorithms } \\
\hline \multirow{3}{*}{ 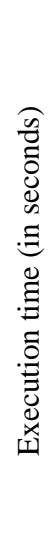 } & Dataset & $\begin{array}{c}\text { Bellman- } \\
\text { Ford }\end{array}$ & $\begin{array}{c}\text { Dijkstra } \\
\text { (Binary } \\
\text { Heap) }\end{array}$ & $\begin{array}{c}\text { Dijkstra } \\
\text { (Edge } \\
\text { based) }\end{array}$ & SPFA & $\begin{array}{c}\text { Proposed } \\
\text { Algorithm }(\mu \\
\quad=1)\end{array}$ & $\begin{array}{c}\text { Proposed } \\
\text { Algorithm }(\mu \\
=2)\end{array}$ & $\begin{array}{c}\text { Proposed } \\
\text { Algorithm }(\mu \\
=3)\end{array}$ \\
\hline & $\begin{array}{c}\text { DIMAC (NE } \\
\text { USA) Distance } \\
\text { Based }\end{array}$ & 103.380 & 5.3560 & 8.0350 & 110.432 & 22.2670 & 22.0450 & 22.8000 \\
\hline & $\begin{array}{c}\text { DIMAC (NE } \\
\text { USA) Time } \\
\text { Based }\end{array}$ & 72.6840 & 6.9900 & 6.7190 & 47.7770 & 20.5570 & 23.1440 & 20.3710 \\
\hline
\end{tabular}




\begin{tabular}{|c|c|c|c|c|c|c|c|c|}
\hline & $\begin{array}{c}\text { Youtube } \\
\text { Communties }\end{array}$ & 3.6290 & 12.2210 & 16.935 & 4.5170 & 13.1500 & 9.8760 & 9.5000 \\
\cline { 2 - 7 } & $\begin{array}{c}\text { Amazon } \\
\text { Purchasing } \\
\text { Network }\end{array}$ & 1.2730 & 3.3360 & 4.3660 & 2.3650 & 5.9210 & 5.1170 & 4.4140 \\
\hline
\end{tabular}

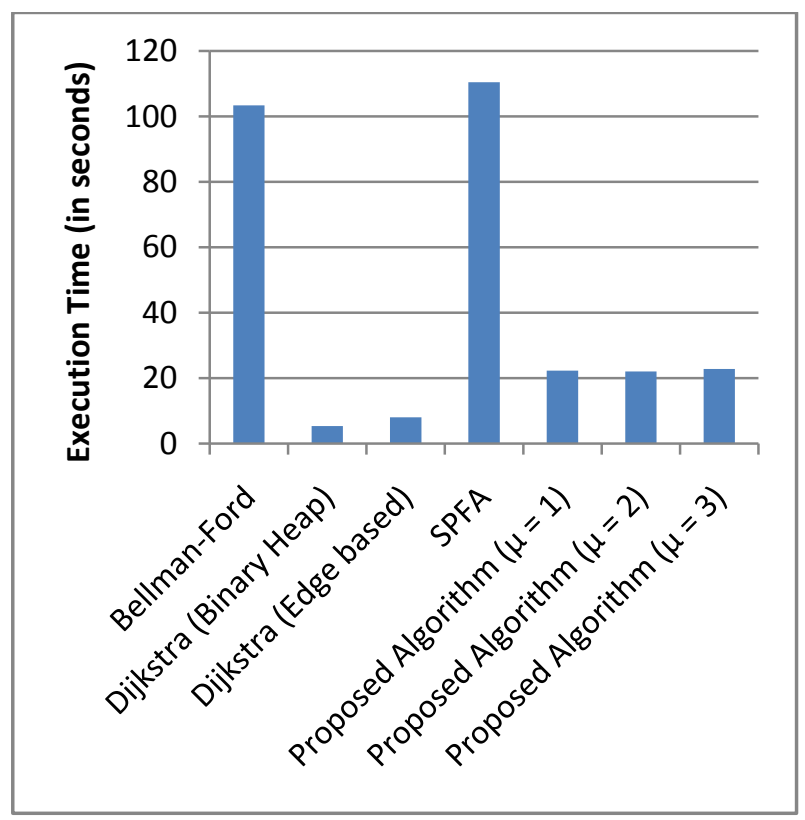

Figure 4: Graph showing execution time on the DIMAC (NE USA) distance based dataset

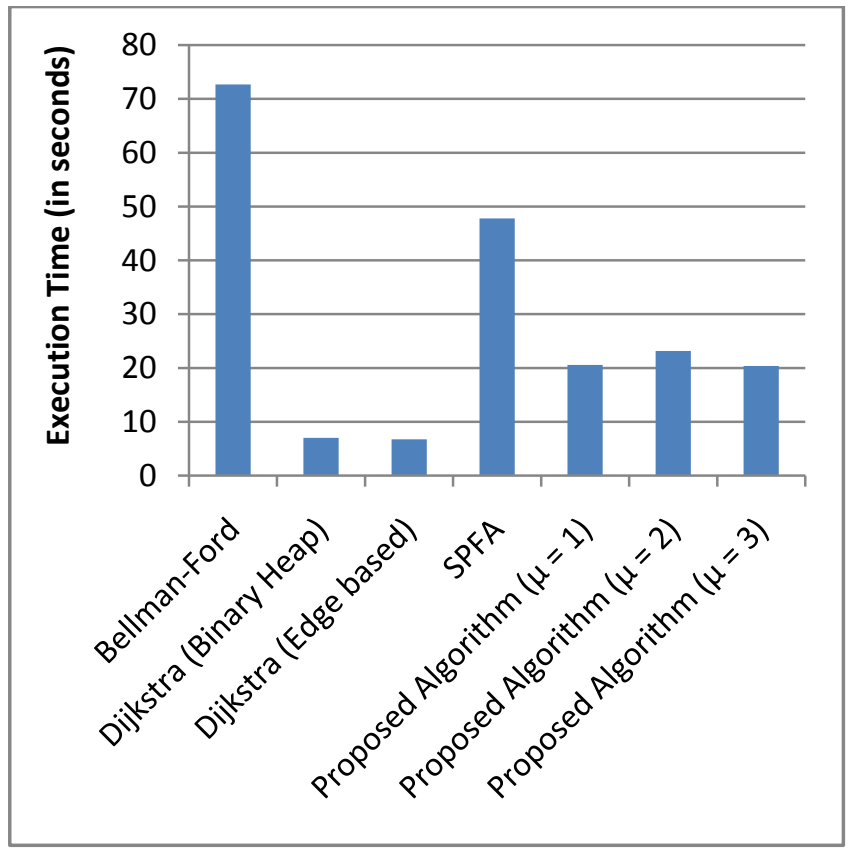

Figure 5: Graph showing execution time on the DIMAC (NE USA) time based dataset

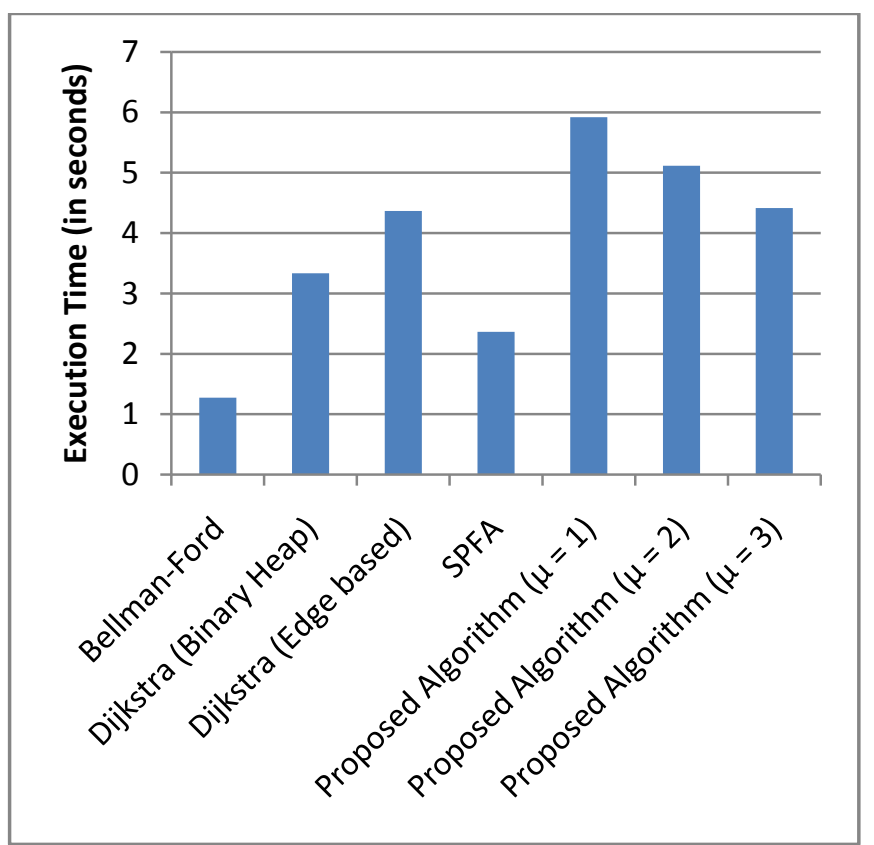

Figure 6: Graph showing execution time on the SNAP Amazon co-purchasing network based dataset

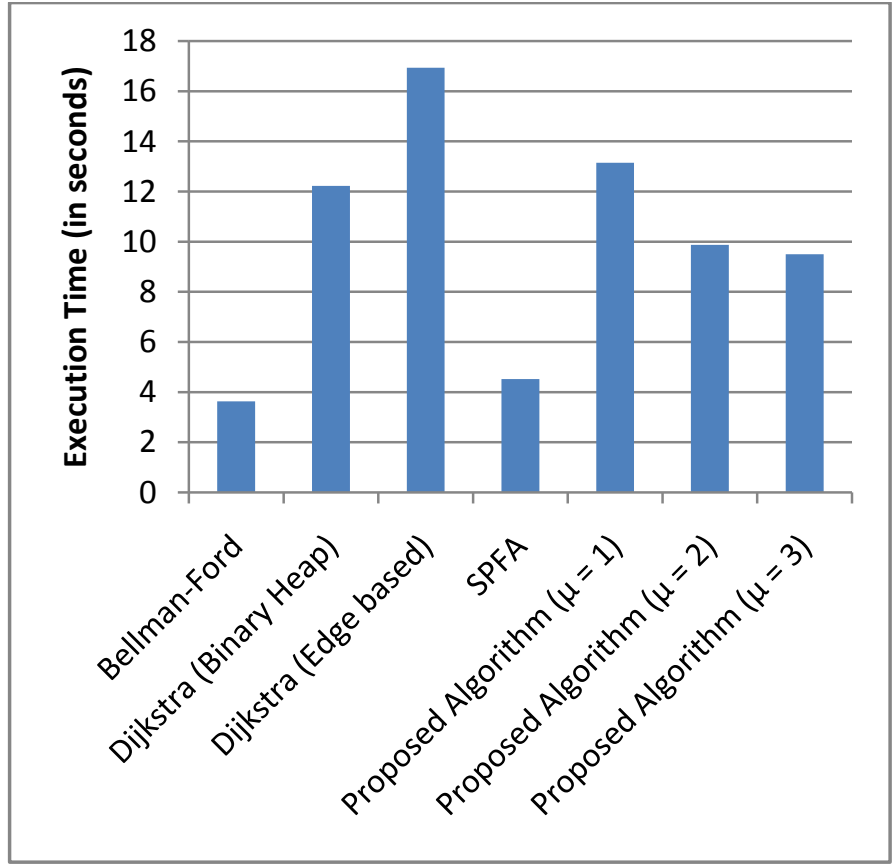

Figure 7: Graph showing execution time on the SNAP YouTube Communities based dataset 


\section{CONCLUSIONS}

It can be seen from the aforementioned analysis that for different datasets, all algorithms under analysis perform differently i.e. certain algorithms perform better as compared to others. For random graphs, SPFA outperforms all the other algorithms while for the general graphs the performance degrades. Performance of algorithms like SPFA highly depends upon the order in which candidate vertices are used to relax other vertices. While processing a vertex, which can be relaxed further increases the time overhead of the algorithm. In case of random graph, this overhead is quite low which make the SPFA performs better in comparison to other algorithms. In case of special graph and benchmarking datasets, this overhead becomes quite high which results in poor performance of SPFA. For all type of graphs the performance of Dijkstra does not depends over the structure of graph, which makes it faster on special graphs. For harnessing the power of both the algorithms our proposed scheme proved to perform better over every kind of graph.

\section{REFERENCES}

[1] ShortestPathProblem,http://en.wikipedia.org/wiki/Shorte st_path_problem

[2] Bellman ford (1958). "On a routing problem". Quarterly of Applied Mathematics. 16: 87-90.Dijkstra, E. W. (1959). "A note on two problems in connexion with graphs". Numerische Mathematik.

[3] Faster Dijkstra on Special Graphs, http://codeforces.com/blog/entry/43508.

[4] Boris V. Cherkassky , Andrew V. Goldberg, Tomasz Radzik, Shortest paths algorithms: theory and experimental evaluation, Mathematical Programming: Series A and B, v.73 n.2, p.129-174, May 31, 1996.

[5] Shortest Path Faster Algorithm, http://codeforces.com/blog/entry/16221.

[6] Prüfer, H. "Neuer Beweis eines Satzes über Permutationen." Arch. Math. Phys. 27, 742-744, 1918.

[7] Construction of Special Graphs for poor performance of SPFA, http://codeforces.com/blog/entry/16221?\#comment211370

[8] 9th DIMACS Implementation Challenge - Shortest Paths, http://www.dis.uniroma1.it/challenge9/download.shtml

[9] Stanford Network Analysis Project, https://snap.stanford.edu/ 